\title{
Conhecimento do enfermeiro sobre o processo de trabalho na Central de Material de Esterilização
}

\author{
Nurses' knowledge about the working process in the Central Sterilization Material
}

Conocimiento del enfermero sobre el proceso de trabajo en la Central de Material de Esterilización

Ilana Maria do Espírito Santo ${ }^{1}$, Patrícia Maria do Espírito Santo², Francisco Lucas de Lima Fontes ${ }^{3 *}$, Maria da Cruz Silva Pessoa Santos ${ }^{3}$, Emilene Paz Freitas ${ }^{4}$, Alzira de Sousa Silva Neta ${ }^{3}$, Janária Oliveira Figueiredo ${ }^{3}$, Francisca Jéssica Abreu da Silva ${ }^{3}$, Léa Jane Gomes Silva ${ }^{1}$, Dulcimar Ribeiro de Matos $^{3}$, Josélia Costa Soares ${ }^{3}$, Maria Aparecida Araújo ${ }^{3}$, Layany Feitosa Pinho ${ }^{3}$, Maria Helena Marcela Gomes Caland ${ }^{3}$, Elizângela Vieira de Araújo ${ }^{4}$.

\section{RESUMO}

Objetivo: Analisar o conhecimento do enfermeiro sobre o processo de trabalho na central de material de esterilização. Métodos: Trata-se de uma revisão integrativa da literatura de natureza exploratória. A busca dos estudos foi realizada na biblioteca virtual Scientific Eletronic Library Online (SciELO) e nas bases de dados Literatura Latino-Americana e do Caribe em Ciências da Saúde (LILACS) e Base de Dados em Enfermagem (BDEnf). Resultados: Foram encontrados 37 artigos e após aplicação dos critérios de inclusão, selecionados 9 artigos para discussão. A literatura relata que o enfermeiro possui déficits de conhecimento advindos de sua formação profissional relacionados a temas do processo de trabalho na Central de Material de Esterilização. Ficou evidente a necessidade de qualificação do enfermeiro que atua neste setor, bem como a da criação de estratégias de educação permanente que contemplem a aquisição e atualização de conhecimentos. Conclusão: As ações deste setor contribuem muito para a minimização de eventos adversos. Contudo, o setor só é lembrado quando algo de errado acontece, revelando a importância de conhecimento sobre o processo de trabalho na Central de Material de Esterilização.

Palavras-chave: Enfermeiro, Centro de esterilização, Educação permanente.

\begin{abstract}
Objective: To analyze nurses' knowledge about the work process of the central sterilization material. Methods: This is an integrative review of the exploratory literature. The search for the studies was carried out in the Scientific Eletronic Library Online (SciELO) virtual library in the Latin American and Caribbean Literature in Health Sciences (LILACS) and Nursing Database (BDEnf) databases. Results: We found 37 articles and after applying the inclusion criteria, we selected 9 articles for discussion. The literature reports that nurses have knowledge deficits arising from their professional training related to work process issues in the Sterilization Material Center. It was evident the need for qualification of nurses working in this sector, as well
\end{abstract}

\footnotetext{
${ }^{1}$ Centro Universitário Uninovafapi. Teresina, Piauí, Brasil.

2 Universidade Estadual do Piauí. Teresina, Piauí, Brasil.

${ }^{3}$ Faculdade UNINASSAU - Campus Redenção. Teresina, Piauí, Brasil. *E-mail: lucasfontesenf@hotmail.com

${ }^{4}$ Centro Universitário Santo Agostinho. Teresina, Piauí, Brasil.
} 
as the creation of permanent education strategies that contemplate the acquisition and updating of knowledge. Conclusion: The actions of this sector contribute much to the minimization of adverse events. However, the industry is only reminded when something goes wrong, hence the importance of knowledge about the work process in the Sterilization Material Center.

Keywords: Nurse, Sterilization, Education continuing.

\section{RESUMEN}

Objetivo: Analizar el conocimiento del enfermero sobre el proceso de trabajo de la central de material de esterilización. Métodos: Se trata de una revisión integrativa de la literatura de naturaleza exploratoria. La investigación de los estudios se realizó en la biblioteca virtual Scientific Electronic Library Online (SciELO) en las bases de datos Literatura Latinoamericana y del Caribe en Ciencias de la Salud (LILACS) y Base de Datos en Enfermería (BDEnf). Resultados: Se encontraron 37 artículos y después de la aplicación de los criterios de inclusión, seleccionados 9 artículos para discusión. La literatura relata que el enfermero posee déficits de conocimiento provenientes de su formación profesional relacionados a temas del proceso de trabajo en la Central de Material de Esterilización. Se evidenció la necesidad de calificación del enfermero que actúa en este sector, así como la de la creación de estrategias de educación permanente que contemplen la adquisición y actualización de conocimientos. Conclusión: Las acciones de este sector contribuyen mucho a la minimización de eventos adversos. Sin embargo, el sector sólo se recuerda cuando ocurre algo malo, de ahí la importancia del conocimiento sobre el proceso de trabajo en la Central de Material de Esterilización.

Palabras-clave: Enfermero, Esterilización, Educación Continua.

\section{INTRODUÇÃO}

As infecções relacionadas à assistência à saúde constituem um grave problema de saúde pública que afeta cerca de 1,5 milhão de pessoas anualmente, devido a sua complexidade e implicações sociais (GIROTI ALB, et al., 2018). Essas infecções são consideradas uma séria ameaça à segurança dos pacientes hospitalizados, o que favorece a elevação das taxas de morbimortalidade, dos custos com procedimentos diagnósticos terapêuticos, além de maior utilização dos leitos de internação (SANTOS AV, et al., 2016).

Os instrumentais utilizados em pacientes internados precisam ser devidamente processados, de modo a impedir que estes tornem-se fômites e transmitam microrganismos a sujeitos sadios (LUCAS TC, et al., 2018). Bugs TV et al. (2013) esclarecem que o correto processamento de utensílios usualmente empregados em hospitais deve relacionar-se à introdução de programas de educação permanente em saúde, com vistas ao alcance de boas práticas por todos os profissionais ocupados nesta atividade, mediante sensibilização, incentivo, construção de ideias e emprego de evidências científicas na vivência profissional para a prevenção de infecções.

Ceccim RB (2005) argumenta que a educação permanente em saúde é fundamental para ruptura com práticas antiquadas dos serviços, bem como atualização contínua dos profissionais, ofertando melhorias, aprendizagens e aperfeiçoamento.

O enfermeiro é o profissional responsável pelas atividades assistenciais ao paciente, norteio da equipe de Enfermagem e administração dos recursos físicos, materiais e humanos (SADE PMC e PERES AM, 2015). A atuação desse profissional fundamenta-se no cuidado direto com o indivíduo e, indireto, no âmbito de busca por melhorias para complementar o embasamento técnico-prático da saúde (REZENDE BC, et al., 2013). Para Taube SAM et al. (2005) o enfermeiro desenvolve também atividades relacionadas ao uso e processamento adequado dos artigos hospitalares. Carvalho VM et al. (2015) ressaltam a importância de os 
profissionais da saúde possuírem conhecimentos relevantes sobre microbiologia, assepsia, desinfecção e esterilização, bem como preparação dos materiais e equipamentos cirúrgicos.

Reiterando a importância das ações adequadas de processamento de materiais, a Resolução do Conselho Federal de Enfermagem no 424 de 2012, normatiza as atribuições dos profissionais de Enfermagem na Central de Material e Esterilização (CME) e em empresas processadoras de produtos para a saúde, estabelecendo ao enfermeiro ações de planejamento, coordenação, execução, supervisão e avaliação de todas as etapas relacionadas ao processamento de produtos para saúde, recepção, limpeza, secagem, avaliação da integridade e da funcionalidade, preparo, desinfecção ou esterilização, armazenamento e distribuição para as unidades consumidoras.

Considerando a importância de conhecimento do enfermeiro acerca do processo de esterilização e desinfecção de artigos hospitalares e reconhecendo o relevante papel deste profissional no controle de infecções, é fundamental que ele mantenha-se em constante atualização sobre o processo de trabalho na CME. Nesse contexto, esse estudo teve como objetivo analisar o conhecimento do enfermeiro sobre o processo de trabalho na Central de Material de Esterilização.

\section{MÉTODOS}

Trata-se de uma revisão integrativa da literatura de natureza exploratória. Este tipo de estudo tem por finalidade reunir e sintetizar os resultados de pesquisas sobre um determinado tema, de maneira sistemática e ordenada, contribuindo desta forma para o aprofundamento do conhecimento do assunto pesquisado (MENDES CDS, et al., 2008).

Para sua confecção seguiu-se seis fases: elaboração da pergunta norteadora, busca ou amostragem na literatura, coleta de dados, análise crítica dos estudos incluídos, discussão dos resultados e apresentação da revisão integrativa (SOUZA MT, et al., 2010).

A questão norteadora foi elaborada por meio da estratégia PICO (P: paciente ou problema, I: intervenção, C: comparação e O: outcomes ou desfecho) conforme segue: "O que mostra a literatura sobre o conhecimento do enfermeiro que atua na Central de Material de Esterilização?".

A pesquisa foi realizada nos meses de agosto e setembro de 2018, utilizando como critérios de inclusão artigos disponíveis na íntegra, de maneira gratuita, em língua portuguesa, publicados entre 2004 e 2018. Como critérios de exclusão optou-se por não utilizar livros, monográficas, dissertações, teses, textos incompletos e artigos que não estavam relacionados à temática estudada.

O levantamento dos artigos na literatura foi realizado por meio de pesquisa na biblioteca virtual Scientific Eletronic Library Online (SciELO) e nas bases de dados Literatura Latino-Americana e do Caribe em Ciências da Saúde (LILACS) e Base de Dados em Enfermagem (BDEnf).

Durante a busca, os descritores foram cruzados entre si com o uso do operador booleano "and", empregouse também o filtro de datas em todas as buscas. Foram utilizados para busca dos artigos os seguintes descritores: "enfermeiro", "centro de esterilização", "educação continuada" e "educação permanente".

Para obtenção dos dados foi elaborado um instrumento para coleta das informações, em que foram coletadas as seguintes variáveis: título dos artigos, nome dos autores, objetivos dos estudos, tipo de pesquisa e principais resultados, tanto a análise quanto a síntese dos dados extraídos dos artigos foram realizadas de forma detalhada, possibilitando examinar, relatar e organizar os dados, com o intuito de reunir o conhecimento produzido sobre o tema explorado na revisão. 


\section{RESULTADOS}

Após a realização das buscas foram encontrados 37 artigos relevantes ao tema estudado, conforme esquema da Figura 1. Para realização desta pesquisa os artigos foram selecionados e submetidos a leitura exploratória, analítica e interpretativa, dentre eles 9 estudos que mais estavam de acordo com tema foram utilizados para construção desta revisão integrativa, sendo: 3 artigos da BDEnf, 5 artigos da SciELO e um artigo da LILACS.

Figura 1 - Processo de busca e seleção dos artigos. Teresina, Piauí, Brasil, 2018.

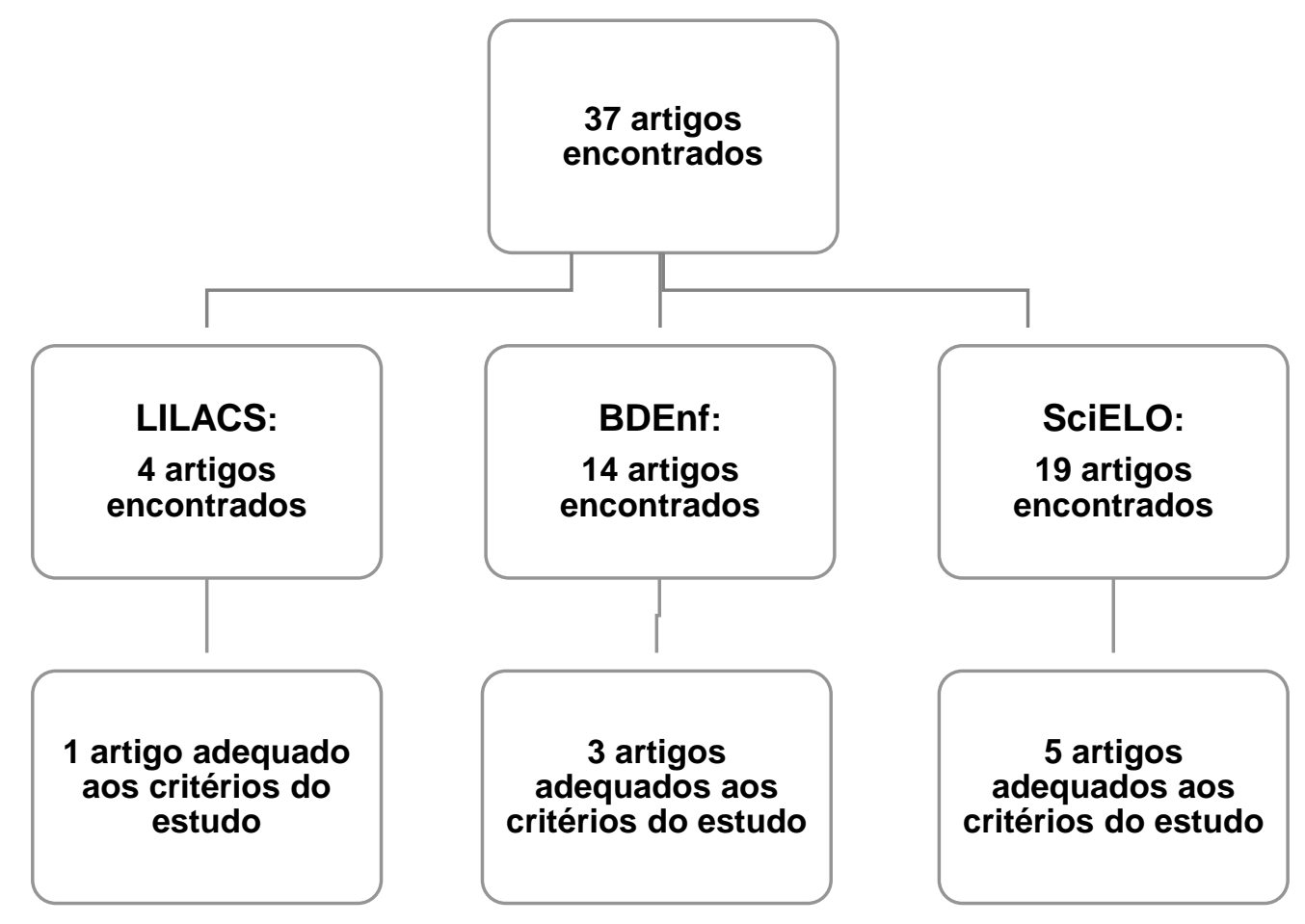

Fonte: Dados da pesquisa, 2018.

Conforme seleção dos artigos utilizados, segue o Quadro 1 com distribuição títulos dos estudos, autores, biblioteca ou base de dados, distribuição cronológica dos anos, tipos de pesquisa e objetivos. 
Quadro 1 - Resultados dos artigos selecionados de acordo com título, autores, biblioteca ou base dados/ano, tipo/abordagem de estudo e conclusões. Teresina, Piauí, Brasil, 2018.

\begin{tabular}{|c|c|c|c|c|}
\hline Título & Autores & $\begin{array}{c}\text { Base / } \\
\text { ano }\end{array}$ & $\begin{array}{l}\text { Tipo de } \\
\text { estudo }\end{array}$ & Conclusão \\
\hline $\begin{array}{l}\text { Enfermagem no } \\
\text { Centro de Material } \\
\text { Esterilizado: a } \\
\text { prática da educação } \\
\text { continuada }\end{array}$ & $\begin{array}{l}\text { SOUZA MCB; } \\
\text { CARIBELLI } \\
\text { MIPF. }\end{array}$ & $\begin{array}{l}\text { SciELO } \\
(2004)\end{array}$ & $\begin{array}{c}\text { Estudo } \\
\text { quantitativo }\end{array}$ & $\begin{array}{l}\text { Observou-se, ainda, a necessidade de um efetivo } \\
\text { serviço de educação continuada com programas } \\
\text { estruturados. }\end{array}$ \\
\hline $\begin{array}{l}\text { O trabalhador sem } \\
\text { formação em } \\
\text { enfermagem } \\
\text { atuando em centro } \\
\text { de material e } \\
\text { esterilização: } \\
\text { desafio para o } \\
\text { enfermeiro }\end{array}$ & $\begin{array}{l}\text { TIPPLE AFV, } \\
\text { SOUZA TR, } \\
\text { BEZERRA } \\
\text { ALQ, et al. }\end{array}$ & $\begin{array}{l}\text { SciELO } \\
(2005)\end{array}$ & $\begin{array}{c}\text { Estudo } \\
\text { quantitativo }\end{array}$ & $\begin{array}{l}\text { Os dados encontrados revelam comprometimento } \\
\text { da qualidade da assistência prestada ao cliente e } \\
\text { evidenciam que há trabalhadores sem formação } \\
\text { específica em Enfermagem, os quais nunca } \\
\text { participaram de programas de educação continuada, } \\
\text { reprocessando artigos odonto-médico-hospitalares. }\end{array}$ \\
\hline $\begin{array}{l}\text { O enfermeiro da } \\
\text { central de material e } \\
\text { esterilização e a } \\
\text { percepção do seu } \\
\text { papel social }\end{array}$ & $\begin{array}{l}\text { BARTOLOMEI } \\
\text { SRT, } \\
\text { LACERDA RA. }\end{array}$ & $\begin{array}{l}\text { BDEnf } \\
(2006)\end{array}$ & $\begin{array}{c}\text { Estudo } \\
\text { qualitativo }\end{array}$ & $\begin{array}{l}\text { Não há tensão entre o seu fazer e a relação, ou não, } \\
\text { com o cuidado pelo enfermeiro. Manifestam } \\
\text { percepção de valor externo negativo do seu trabalho, } \\
\text { suficiente para geração de tensão e desconforto. }\end{array}$ \\
\hline $\begin{array}{l}\text { Processo de } \\
\text { trabalho do } \\
\text { enfermeiro na } \\
\text { central de material e } \\
\text { esterilização: } \\
\text { Percepção de } \\
\text { estudantes de } \\
\text { graduação em } \\
\text { enfermagem }\end{array}$ & $\begin{array}{l}\text { TAUBE SAM, } \\
\text { LABRONICI } \\
\text { LM, MAFTUM } \\
\text { MA et al. }\end{array}$ & $\begin{array}{l}\text { BDEnf } \\
(2008)\end{array}$ & $\begin{array}{c}\text { Estudo } \\
\text { qualitativo }\end{array}$ & $\begin{array}{l}\text { Sugere-se que os cursos de graduação abordem } \\
\text { temas relativos ao processo de trabalho na CME que } \\
\text { demonstrem a amplitude do campo de atuação do } \\
\text { enfermeiro, para que os estudantes visualizem e } \\
\text { valorizem o saber-fazer desse profissional no } \\
\text { processo de cuidar em saúde. }\end{array}$ \\
\hline $\begin{array}{l}\text { Enfermagem no } \\
\text { processo de } \\
\text { esterilização de } \\
\text { materiais }\end{array}$ & $\begin{array}{l}\text { OURIQUES } \\
\text { CM, } \\
\text { MACHADO } \\
\text { ME. }\end{array}$ & $\begin{array}{l}\text { SciELO } \\
(2013)\end{array}$ & $\begin{array}{c}\text { Estudo } \\
\text { qualitativo }\end{array}$ & $\begin{array}{l}\text { Os trabalhadores têm conhecimento incipiente das } \\
\text { etapas do processo de esterilização, encontrando na } \\
\text { educação permanente em saúde a possibilidade da } \\
\text { redução de falhas no processo de trabalho, assim } \\
\text { como da adoção de uma postura reflexiva acerca da } \\
\text { importância de seu trabalho. }\end{array}$ \\
\hline $\begin{array}{l}\text { Necessidades de } \\
\text { aprendizagem de } \\
\text { enfermeiros sobre } \\
\text { processos de } \\
\text { esterilização }\end{array}$ & $\begin{array}{l}\text { PADOVEZE } \\
\text { MC, } \\
\text { FIGUEIREDO } \\
\text { RM, PELAES } \\
\text { CES et al. }\end{array}$ & $\begin{array}{l}\text { BDEnf } \\
(2013)\end{array}$ & $\begin{array}{c}\text { Estudo } \\
\text { quantitativo }\end{array}$ & $\begin{array}{l}\text { Os principais tópicos de aprendizagem indicados } \\
\text { pelos participantes foram referentes à validação, } \\
\text { monitorização e microbiologia da esterilização. } \\
\text { Evidenciou-se a necessidade de produzir } \\
\text { treinamentos que ultrapassem os limites superficiais } \\
\text { do conhecimento no que tange aos processos de } \\
\text { esterilização. }\end{array}$ \\
\hline $\begin{array}{l}\text { Atividades do } \\
\text { enfermeiro de centro } \\
\text { de material e } \\
\text { esterilização em } \\
\text { instituições } \\
\text { hospitalares }\end{array}$ & $\begin{array}{l}\text { GIL RF, } \\
\text { CAMELO SH, } \\
\text { LAUS AM. }\end{array}$ & $\begin{array}{l}\text { SciELO } \\
(2013)\end{array}$ & $\begin{array}{c}\text { Estudo } \\
\text { quantitativo }\end{array}$ & $\begin{array}{l}\text { Evidencia-se a necessidade de incorporar novas } \\
\text { questões sobre o futuro do trabalho no Centro de } \\
\text { Material e Esterilização, sugerindo o } \\
\text { desenvolvimento de novas pesquisas na área. }\end{array}$ \\
\hline $\begin{array}{l}\text { Formação do } \\
\text { enfermeiro para } \\
\text { atuar na central de } \\
\text { esterilização }\end{array}$ & $\begin{array}{l}\text { LUCON SMR, } \\
\text { BRACCIALLI } \\
\text { LAD, PIROLO } \\
\text { SM et al. }\end{array}$ & $\begin{array}{l}\text { LILACS } \\
(2017)\end{array}$ & $\begin{array}{l}\text { Estudo } \\
\text { qualitativo }\end{array}$ & $\begin{array}{l}\text { Independente da proposta curricular das instituições } \\
\text { de ensino, os enfermeiros tiveram dificuldades para } \\
\text { o aprofundamento quanto ao tema proposto, mas } \\
\text { colaboraram com sugestões de transformações das } \\
\text { práticas de ensino e aprendizagem para atuação dos } \\
\text { enfermeiros na CME e que essa formação possa ser } \\
\text { repensada mais adequadamente. }\end{array}$ \\
\hline $\begin{array}{l}\text { Estratégias que } \\
\text { contribuem para a } \\
\text { visibilidade do } \\
\text { trabalho do } \\
\text { enfermeiro na } \\
\text { central de material e } \\
\text { esterilização }\end{array}$ & $\begin{array}{l}\text { SANCHEZ } \\
\text { ML, SILVEIRA } \\
\text { RS, } \\
\text { FIGUEIREDO } \\
\text { PP et al. }\end{array}$ & $\begin{array}{c}\text { SciELO } \\
(2018)\end{array}$ & $\begin{array}{c}\text { Estudo } \\
\text { qualitativo }\end{array}$ & $\begin{array}{l}\text { Evidencia-se a necessidade de engajamento dos } \\
\text { próprios enfermeiros da Central de Material e } \\
\text { Esterilização para se fazerem perceber e } \\
\text { modificarem a imagem do setor e a maneira como a } \\
\text { administração das instituições de saúde vem lidando } \\
\text { com os avanços do processamento dos artigos } \\
\text { médico-cirúrgicos. }\end{array}$ \\
\hline
\end{tabular}

Fonte: Dados da pesquisa, 2018. 


\section{DISCUSSÃO}

A CME é uma unidade de apoio técnico dentro do estabelecimento de saúde destinada a receber material considerado sujo e contaminado para descontaminá-lo, prepará-lo e esterilizá-lo, bem como, preparar e esterilizar também as roupas limpas advindas da lavanderia e armazenar esses artigos para futura distribuição (GIL RF, et al., 2013).

A atuação do enfermeiro na CME requer conhecimentos específicos sobre a diversidade de equipamentos, artigos e instrumentos cirúrgicos, assim como a forma correta de processá-los. Configura-se como uma área de atuação da Enfermagem, cujo objetivo é garantir produtos seguros para a assistência ao paciente (LUCON SMR, et al., 2017). A articulação de conhecimentos constitui estratégias de trabalho da Enfermagem. A atuação do enfermeiro neste setor, área eminentemente administrativa, relaciona-se à organização do ambiente, por meio do cuidado indireto (BARTOLOMEI RST e LACERDA RA, 2006).

Suas atividades são norteadas em três eixos de organização: direção da unidade, atuação técnicaadministrativa e participação no dimensionamento de pessoal. Entre as práticas de direção encontram-se: garantia de materiais aos setores consumidores, elaboração de relatórios, participação em comissões institucionais e gerenciamento das atividades de Enfermagem. A atuação técnica-administrativa atrela-se ao planejamento, coordenação e desenvolvimento das rotinas de preparo dos artigos, gerenciamento de equipamentos consignados, garantia de conservação dos materiais e elaboração da passagem de plantão. Quanto ao dimensionamento de pessoal, o enfermeiro deve garantir participação em planejamentos de prevenção e controle de eventos adversos, execução de capacitações contínuas, elaboração de escalas de trabalho e triagem e seleção de pessoal (TAUBE SAM, et al., 2008).

O estudo de Ouriques CM e Machado ME (2013) revelou o desconhecimento dos profissionais de Enfermagem acerca das funções que deveriam exercer efetivamente no setor, o que denota para a relevância de educação permanente em saúde. Ações de construção e atualização de conhecimentos influenciam na redução de falhas na dinâmica de trabalho. O enfermeiro, profissional responsável pela CME, participa do processo de capacitação, educação e avaliação do desempenho de profissionais da unidade.

Assegurar a qualidade de processamento dos produtos para a saúde é relevante, pois a distribuição dos materiais processados interfere positivamente ou não no processo saúde-doença, ao passo que más práticas durante o manejo desses utensílios na CME facilitam a ocorrência de artigos mal processados, o que gera eventos adversos, como o surgimento de infecções.

Para se evitar tais eventos, são necessárias capacitações dos profissionais da CME para a classificação dos artigos; entendimento dos conceitos de microbiologia; noções sobre a condução de produtos contaminados; atualização sobre os processos de limpeza, desinfecção, preparo, inspeção, acondicionamento, recursos de barreira estéril (embalagens), esterilização, desempenho dos equipamentos, inspeção de processos por indicadores químicos, biológicos e físicos, rastreabilidade, armazenamento, distribuição e manutenção da esterilidade do produto (GIL RF, et al., 2013).

Tipple AFV et al. (2005) enfatizam a necessidade de aperfeiçoamento contínuo do trabalho na CME e de seus membros, abandonando o voluntarismo e o consequente amadorismo. Para os autores, o mercado de trabalho está desejoso de profissionais competentes, que saibam agir, tomar decisões e usar da criatividade; é necessário priorizar tais qualidades no momento do recrutamento de profissionais.

Torna-se relevante a conscientização da importância da CME no contexto hospitalar, com vistas a execução de uma assistência eficiente ao paciente, sendo fundamental um efetivo programa de educação em serviço, uma vez que a qualificação do enfermeiro relaciona-se diretamente à qualidade de área em que atua (SOUZA MCB e CARIBELLI MIPF, 2004).

REAS/EJCH | Vol. Sup. 20 | e403 | DOI: https://doi.org/10.25248/reas.e403.2019 Página 6 de 8 
Segundo Sanchez et al. (2018), os encargos exercidos pelo enfermeiro na CME por vezes são imperceptíveis. Mesmo sendo visto como significativo por outros profissionais, grande parte ignora a ampla gama de atividades desempenhadas pelo setor e a necessidade de conhecimento singular para atuação na área. Em seu estudo, os autores também evidenciaram a importância da educação permanente voltada às temáticas de processamento de materiais. A educação permanente desenvolve a capacidade do trabalhador para o aprender a aprender, para a tomada de consciência acerca de suas necessidades e aperfeiçoa as habilidades técnicas necessárias ao trabalho na CME.

O desconhecimento sobre o processo de trabalho e do papel do enfermeiro na CME, evidencia a necessidade de abordagem, pelos cursos de graduação em Enfermagem a temas relativos ao processo de trabalho no setor, para que os estudantes possam conhecer e valorizar o saber-fazer desse profissional no processo de cuidar em saúde (TAUBE SAM, et al., 2008).

O conteúdo específico de CME, em algumas grades de Enfermagem, é abordado na disciplina de Centro Cirúrgico, entretanto esse ensino é insuficiente, sendo demasiadamente vago e curto, o que se transforma em empecilho, já que esse assunto deveria ser abordado de forma mais abrangente durante toda a graduação (LUCON SMR, et al., 2017).

A complexidade envolvendo as atividades de processamento de materiais associada à falta de abordagem na graduação, exige que os enfermeiros busquem processos educativos que aumentem sua capacitação profissional. Essa busca por metodologias novas de ensino alteram o paradigma na formação dos enfermeiros, respondendo assim, aos problemas de saúde da população (PADOVEZE et al., 2013). É imprescindível que esses profissionais saiam de sua graduação com os conhecimentos básicos sobre CME (LUCON SMR, et al., 2017).

\section{CONCLUSÃO}

As atividades desempenhadas na CME constituem-se de grande relevância na prevenção e controle das infecções relacionadas à assistência à saúde. As ações deste setor contribuem muito para a minimização de eventos adversos. Contudo, o setor só é lembrado quando algo de errado acontece, daí a importância de conhecimento sobre o processo de trabalho na CME. Observa-se, portanto, lacunas sobre a dinâmica de trabalho no setor. É de fundamental importância estimular o sujeito, ainda na graduação, a adquirir conhecimentos e habilidades representativas nessa área. Para superar tal lógica, propõem-se práticas educativas que valorizem o preparo e a motivação dos estudantes, formando profissionais críticos, motivados, criativos, flexíveis e comprometidos com o objetivo de sua atuação. Os fatores que favorecem essa lacuna escapam do âmbito desta revisão, no entanto, parece claro que, o fato de os processos formativos da Enfermagem abordarem a CME superficialmente pode provocar nos estudantes certo distanciamento e 0 receio do desconhecido pode levá-los a não se aprofundarem na área. Frente ao obstáculo evidenciado e aos resultados apontados nos artigos incluídos nesta revisão integrativa, sugere-se maiores estudos que discutam sobre o processo educacional do enfermeiro atuante na CME e suas ações no setor.

\section{REFERÊNCIAS}

1. BARTOLOMEI SRT, LACERDA RA. O enfermeiro da central de material e esterilização e a percepção do seu papel social. Revista Gaúcha de Enfermagem, 2006; 27(2): 258-265.

2. BUGS TV, RIGO DFH, BOHRER CD et al. Perfil da equipe de Enfermagem e percepções do trabalho realizado em uma central de materiais. Revista Mineira de Enfermagem, 2017; 21(e-996): 1-8.

3. CARVALHO VM, MOURA MEB, BATISTA OMA et al. Conhecimento dos profissionais de enfermagem sobre fatores de risco relacionados à infecção de sítio cirúrgico. Revista Interdisciplinar, 2015; 8(3): 1-11. 
4. CECCIM RB. Educação Permanente em Saúde: descentralização e disseminação de capacidade pedagógica na saúde. Ciência \& saúde coletiva, 2005; 10(4): 975-986.

5. CONSELHO FEDERAL DE ENFERMAGEM (COFEN). Resolução oㅜ 424, de 19 de abril de 2012. COFEN, 2012.

6. GIL RF, CAMELO SH, LAUS AM. Atividades do enfermeiro de centro de material e esterilização em instituições hospitalares. Texto \& Contexto Enfermagem, 2013; 22(4): 927-934.

7. GIROTI ALB, FERREIRA AM, RIGOTTI MA et al. Programas de Controle de Infecção Hospitalar: avaliação de indicadores de estrutura e processo. Revista da Escola de Enfermagem da USP, 2018; 52(e03364): 1-7.

8. LUCAS TC, SOUZA MX, GUEDES HM et al. Identificação de deteriorações físicas e químicas nos instrumentais cirúrgicos após reprocessamentos. Revista de Enfermagem do Centro-Oeste Mineiro, 2018; 8(e1926): 1-8.

9. LUCON SMR, BRACCIALLI LAD, PIROLO SM et al. Formação do enfermeiro para atuar na central de esterilização. Revista SOBECC, 2017; 22(2): 90-97.

10. MENDES CDS, SILVEIRA RCCP, GALVÃO CM. Revisão integrativa: método de pesquisa para a incorporação de evidências na saúde e na enfermagem. Texto \& Contexto Enfermagem, 2008; 17(4): 758-764.

11. OURIQUES CM, MACHADO ME. Enfermagem no processo de esterilização de materiais. Texto \& Contexto Enfermagem, 2013; 22(3): 695-703.

12. PADOVEZE MC, FIGUEIREDO RM, PELAES, CES et al. Necessidades de aprendizagem de enfermeiros sobre processos de esterilização. Revista SOBECC, 2013; 18(3): 23-29.

13. REZENDE BC, VASCONCELOS RMA, LIMA SS et al. Dificuldades enfrentadas pelos enfermeiros na prática da liderança em enfermagem: uma revisão da literatura. Revista Eletrônica Gestão \& Saúde, 2013; 4(2): 2273-2288.

14. SADE PMC, PERES AM. Desenvolvimento de competências gerenciais do enfermeiro: diretriz para serviços de educação permanente. Revista da Escola de Enfermagem da USP, 2015; 49(6): 991-998.

15. SANCHEZ ML, SILVEIRA RS, FIGUEIREDO PP et al. Estratégias que contribuem para a visibilidade do trabalho do enfermeiro na central de material e esterilização. Texto \& Contexto Enfermagem, 2018; 27(1): e6530015.

16. SANTOS AV, SILVA MRP, CARVALHO MM et al. Perfil das infecções hospitalares nas unidades de terapia intensiva de um hospital de urgência. Revista de Enfermagem da UFPE, 2016; 10(Sup.1): 194-201.

17. SOUZA MCB, CARIBELLI MIPF. Enfermagem no centro de material esterilizado: a prática da educação continuada. Revista Latino-Americana de Enfermagem, 2004; 12(5): 767-774.

18. SOUZA MT, SILVA MD, CARVALHO R. Revisão integrativa: o que é e como fazer. Einstein, 2010; 8(1pt1): $102-106$.

19. TAUBE SAM, LABRONICI LM, MAFTUM MA et al. Processo de trabalho do enfermeiro na central de material e esterilização: percepção de estudantes de graduação em enfermagem. Ciência, Cuidado e Saúde, 2008; 7(4): 558564 .

20. TAUBE SAM, ZAGONEL IPS, MEIER MJ. Um marco conceitual ao trabalho da enfermagem na central de material e esterilização. Cogitare enfermagem, 2005; 10(2): 76-83.

21. TIPPLE AFV, SOUZA TR, BEZERRA ALQ et al. O trabalhador sem formação em enfermagem atuando em centro de material e esterilização: desafio para o enfermeiro. Revista da Escola de Enfermagem da USP, 2005; 39(2): $173-180$. 